\title{
Donepezil slows decline in daily life activities in people with moderate to severe Alzheimer's disease and alleviates caregiver burden
}

Feldman H, Gauthier S, Hecker J, et al. Efficacy of donepezil on maintenance of activities of daily living in patients with moderate to severe Alzheimer's disease and the effect on caregiver burden. J Am Geriatr Soc 2003;51:737-44.

What are the effects of donepezil on activities of daily living, and social functioning in people with moderate to severe Alzheimer's disease, and what are the effects on caregiver burden?

METHODS

口

Design: Randomised controlled trial.

Allocation: Concealed.

Blinding: Double blind.

Follow up period: 24 weeks.

Setting: 32 centres in Canada (22), Australia (6) and France (4)

Patients: 290 people with moderate to severe Alzheimer's disease (assessed using National Institute of Neurological and Communicative Disorders and Strokes-Alzheimer's Disease and Related Disorders Association criteria), with a committed caregiver, living in the community or in assisted living facilities. Participants had standardised Mini-Mental State Examination (sMMSE) scores of 5 to 17 ; mean age 73 years (donepezil group), and 74 years (placebo group). Exclusions: requiring 24 hour skilled nursing care; confinement to wheelchair; and taking medications with cholinomimetic or anticholinergic effects.

Participants taking psychoactive medications had to have been on a stable dose for at least 4 weeks and remained on this dose for at least 4 weeks after the start of the trial. Participants could be initiated on psychoactive medications only affer 4 weeks in the trial.

$\mathbf{R}_{\mathbf{X}}$

Intervention: Placebo or donepezil once daily for 24 weeks. Participants in the active treatment group received $5 \mathrm{mg} /$ day donepezil for 28 days before increasing to $10 \mathrm{mg} /$ day for the remainder of the trial if tolerated. Caregivers maintained contact with the participant for at least 8 hours three times a week and accompanied them to study visits. Caregivers were preferably non-paid carers (ie relatives, friends, or neighbours) and were requested to keep a caregiver diary and complete caregiver ratings before study visits.

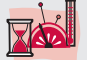

Outcomes: Ability to perform instrumental activities of daily living (IADLs) and basic activities of daily living (BADLs) as assessed by the Disability Assessment for Dementia scale (DAD caregiver-rated scale), a modified IADL scale (modified to include selected items from the Alzheimer's Disease Functional Assessment and Change Scale), and a modified Physical SelfMaintenance Scale (PSMS, modified to include important BADL activities). Caregiver stress was measured on a modified Caregiver Stress Scale (CSS).

Patient follow up: $85 \%$

\section{MAIN RESULTS}

Donepezil slowed decline in participants' ability in ADLs compared with placebo (at week 24 mean difference in score: 6.83 for modified IADL score, $\mathrm{p}<0.0001 ; 1.32$ for modified PSMS score, $\mathrm{p}=0.0015$;

For correspondence: Howard Feldman, Division of Neurology, UBC Hospital, Westbrook Mall, Vancouver, Canada; hfeldman@interchange.ubc.ca

Sources of funding: Pfizer Inc, New York and Eisai Inc, Teaneck, New Jersey.
8.24 for DAD score, $\mathrm{p}<0.0001$; all figures based on least squares mean change from baseline, last observation carried forward). Caregivers for the donepezil group were less stressed than caregivers for the placebo group (at week 24 mean difference in CSS score: 1.82, significance not stated for this comparison). Caregivers for the donepezil group reported spending significantly less time assisting participants with ADL activities than caregivers for the placebo group (at week 24 mean difference in time: $52.4 \mathrm{~min} /$ day, $\mathrm{p}=0.004$ ).

\section{CONCLUSIONS}

Donepezil significantly slowed decline in ability to perform IADLs and BADLs in people with moderate to severe Alzheimer's disease. Caregivers for the donepezil group spent significantly less time assisting them with ADL activities and were less stressed.

\section{NOTES}

Authors note that all measures of ADL ability used in this study were based on caregiver assessment. The validity of these subjective ratings has not been tested.

\section{Commentary}

M ost RCTs of "anti-dementia" drugs for Alzheimer's disease have focused on cognitive functioning in people with mild to moderate dementia. Feldman et al's study adds to the evidence already available for rivastigmine, ${ }^{1}$ and is of importance because it demonstrates the benefits of donepezil on activities of daily living (ADL) in people with moderate to severe dementia, and quantifies the associated reduction in caregiver burden.

Evidence for the benefits of "anti-dementia" drugs for people with moderate to severe dementia is of particular interest to families and practitioners in the UK where national guidelines have recommended that treatment cease after MMSE scores drop below $12 .^{2}$ The reduction in caregiver burden will also be of interest to policy makers, given the known association between burden and admission to paid care facilities.

Feldman et al have overcome the challenges of studying people with low functional ability and a deteriorating illness by using measures of basic ADL and the associated time spent by caregivers. However, excluding night-time hours from the analysis may have left significant ADL support time unmeasured given that dementia and donepezil have been associated with increased sleep disturbances. ${ }^{3}$ Nighttime disturbance has a great impact on family carers' quality of life, but could not be measured due to some caregiver participants being paid staff for patients in assisted care facilities.

This study adds significant evidence for the use of donepezil for people with moderate to severe dementia and demonstrates the associated reduction in caregiver burden. Caregiver involvement in future research and clinical decision making could usefully identify how much change is needed to make a meaningful difference to quality of life.

Geraldine Hancock DCIPs, PhD and Georgina Charlesworth ClinPsyD University College London, London, UK

1 Potki SG, Anand R, Hartman R, et al. Impact of Alzheimer's disease and rivastigmine treatment on activities of daily living over the course of mild to moderately severe disease. Prog Neuropsychopharmacol Biol Psychiatry 2002;26:713-20

2 National Institute for Clinical Excellence (NICE). Guidance on the use of Donepezil, Rivastigmine, and Galantamine for the treatment of Alzheimer's Disease. Technology Appraisal Guidance No 19. London: Department of Health, 2001.

3 Stahl SM, Markowitz JS, Gutterman EM, et al. Co-use of donepezil and hypnotics among Alzheimer's disease patients living in the community. $J$ Clin Psychiatry 2003;64:466-72. 